Pathologe 2008 · [Suppl 2] 29:250-254

DOI 10.1007/s00292-008-1093-0

Online publiziert: 9. Oktober 2008

(c) Springer Medizin Verlag 2008

P. Hufnagl $\cdot$ K. Schlüns

Institut für Pathologie, Charité-Universitätsmedizin Berlin, Campus Mitte

\title{
Virtuelle Mikroskopie und Routinediagnostik
}

\section{Ein Diskussionspapier}

durch die nachstehenden Fakten charakterisiert:

Die virtuelle Mikroskopie (VM) war nicht ohne Grund ein zentrales Thema der Jahrestagung der Deutschen Gesellschaft für Pathologie (DBP) 2008. Aus einem Randthema strebt die VM in das Zentrum der Pathologie - in die alltägliche Diagnostik.

Wird sie dort ankommen? Wenn ja, warum und wann, und welche Auswirkungen wird das auf das Fachgebiet haben? Diese Fragen werden zurzeit noch konträr diskutiert. Parallel dazu verläuft eine schnelle technische und methodische Weiterentwicklung der VM sowie eine stetig voranschreitende Nutzung in der Ausund Weiterbildung, der Qualitätssicherung und in der Forschung [1-6].

Wo liegt das Potenzial der VM in der Routine? Wo liegen die Grenzen? Und mit welchen Zeiträumen bei der Einführung muss man rechnen? Hat die Methodik Auswirkungen auf die Tätigkeit des $\mathrm{Pa}$ thologen allgemein? Wie können die Pathologen die VM zum Nutzen des Fachgebiets und seiner Stellung in der Krankenversorgung einsetzen?

\section{Virtuelle Mikroskopie als Methode}

Unter VM versteht man das Erstellen von kompletten elektronischen Abbildern mikroskopischer Präparate und deren analog zum Mikroskopieren erfolgende Visualisierung über den Computer. Die beim Einscannen der Objektträger entstehenden Bilder nennt man „Whole Slide Images" (WSI; [7]). Wesentliche methodische Unterschiede zwischen konventioneller Mikroskopie und VM werden
1. Die Trennung von Präparat (WSI) und Mikroskop (Scanner) bei der Mikroskopie.

2. Ein WSI ist eine elektronische Datei.

3. Ein WSI ist ein digitales Bild.

Diese zunächst banal anmutenden Fakten haben eine Reihe von Konsequenzen.

$Z u$ 1.: Die Trennung von Präparat und Mikroskop erlaubt es dem Pathologen nicht mehr, auf die Abbildung eines histologischen Präparates beim Mikroskopieren direkt Einfluss zu nehmen. So kann er zum Zeitpunkt seiner Untersuchung am WSI etwa keinen Polarisationsfilter einsetzen, um z. B. Amyloid nachzuweisen. Er muss sich heute in der Regel auch mit der Fokusebene begnügen, die eingescannt wurde, und kann nicht durch Drehen der Mikrometerschraube in Z-Richtung das Präparat durchfokussieren, obwohl die Entwicklung bereits dahin geht [8].

$\mathrm{Zu}$ 2.: Eine Datei kann nur in Verbindung mit Computern genutzt werden. Dateien können kopiert, verändert und gelöscht, aber auch über das Internet für andere Nutzer bereitgestellt werden. Dateien erfordern eine elektronische Speicherung. Speichermedien veralten, und die gespeicherten Daten müssen auf neue Medien übertragen werden. Die Unversehrtheit von Dateien kann beispielsweise durch eine digitale Signatur sichergestellt werden (digitaler Arztausweis).

Zu 3.: Digitale Bilder können mit Bildverarbeitungsprogrammen verändert werden. Dies kann sowohl für Zwecke der Vi- sualisierung (um z. B. verschiedene Färbungen eines Präparates optisch übereinander liegend darzustellen) als auch der quantitativen Analyse (Bestimmung des Anteils positiv markierter Zellen) verwendet werden [9].

Im Gegensatz zu histologischen Präparaten altern WSI nicht. Jeder Lehrende kann an den WSI eigene Markierungen und Textbezüge anbringen. Allgemein bietet die VM für die Lehre ein faszinierendes, ausgesprochen universelles und effektives Handwerkszeug. Dies wird durch die Entstehung immer neuer universitärer Kurse und Programme unterstrichen, die teilweise über das Internet frei zugänglich, teilweise im Tauschverfahren elektronischer Lehrangebote zwischen Universitäten angeboten werden. Parallel etabliert sich zunehmend ein Angebot kommerzieller Produkte.

In der Wissenschaft und in der industriellen Forschung etabliert sich die VM als effektives Hilfsmittel. Der mit Abstand größte Teil von Präparatescannern ist in Unternehmen der Pharmaindustrie im Einsatz. So wird beispielsweise die quantitative Auswertung von „Tissue Microarrays" (TMAs) durch den Übergang zu WSI wesentlich vereinfacht und einer Automatisierung zugänglich. Allein die Orientierung in einem komplexen TMA und die jederzeit sichere Dokumentation des Arbeitsstandes spart bereits Zeit und erhöht die Qualität und Reproduzierbarkeit der Auswertung [9]. 


\section{Virtuelle Mikroskopie als Alternative zur konventionellen Mikroskopie?}

In der digitalen Radiologie war allein die Betrachtungsmöglichkeit einer Computertomographie am PC ein eindeutiger Fortschritt. Im Gegensatz dazu muss die Einführung der VM in der alltäglichen Diagnostik einen klaren Mehrwert gegenüber der konventionellen Mikroskopie erbringen. Dieser Mehrwert kann sich in zeitlichen und finanziellen Einsparungen und/oder erweiterter Funktionalität ausdrücken.

Für die Einführung der VM in die Routine müssen deshalb eine Reihe von Anforderungen erfüllt sein:

\section{Wirtschaftlichkeit}

Die Einführung der VM muss klare wirtschaftliche Vorteile bringen.

Man benötigt im Wesentlichen Präparatescanner, Speicher und eine VM-Software. Nicht zu vernachlässigende Kosten entstehen durch die notwendige Integration in das Gesamtsystem (s. unten). Allein die Einsparung des Glasarchivs (unter der Voraussetzung, dass dies bereits rechtlich möglich wäre) oder der Wege zum Archiv reichen nicht aus, um die Anschaffungskosten zu legitimieren.

Soll die VM dagegen eingesetzt werden, um mehrere Standorte einer Einrichtung miteinander zu verbinden, so sieht die Bilanz bereits ganz anders aus, und die Investition kann allein durch den Wegfall von Wegezeiten gerechtfertigt sein. Ganz allgemein sieht die Wirtschaftlichkeitsrechnung für unterschiedlich große, strukturierte und aufgestellte Pathologien durchaus sehr verschieden aus.

\section{Bildqualität}

Die Bildqualität muss höchsten Ansprüchen genügen.

Zurzeit haben Pathologen häufig das Gefühl, dass WSI im Vergleich zum direkten Mikroskopbild eine schlechtere Qualität haben.

Die gleiche Situation gab es in der digitalen Radiologie auch. Am Anfang der Entwicklung waren die digitalen Bilder schlechter, schon aufgrund der da- mals aus Kapazitätsgründen notwendigen Beschränkung der Auflösung. Die gesamte Abbildungskette („Scanning“, "Streaming“, „Viewing“) muss in Hardware und Software optimiert werden. Für WSI wurde der aktuelle Status in einigen Studien untersucht und kein gravierender, diagnostisch relevanter Qualitätsverlust festgestellt (Übersicht in [10]). De facto wird sich die Qualität der WSI immer weiter verbessern, und am Ende wird sie über der mit dem konventionell genutzten Lichtmikroskop erreichbaren Qualität liegen. Als Beispiel hierfür seien computerbasierte Optimierungen der Fokussierung genannt: Auf der Grundlage eines Z-Stapels von WSI eines Präparates kann ein konfokaler WSI berechnet werden [11]. Genauso kann eine Superposition berechnet werden, die z. B. alle Nukleoli eines Zellkernes gleichzeitig visualisiert, ohne dass die „Fokusschraube“ betätigt werden muss.

\section{Geschwindigkeit und Bedienkomfort}

Virtuelle Mikroskope müssen einfach bedienbar und schnell sein.

Allgemein erscheint VM langsamer als konventionelles Mikroskopieren, wenn man etwa an das schnelle Verschieben eines Präparates mit der Hand ohne Objektträgerhalter denkt. Auch ist die Bedienung virtueller Mikroskope mit der PCMaus nicht zufrieden stellend möglich.

Hier besteht ein Handlungsbedarf für die Entwickler virtueller Mikroskope, und es wird bereits nach Alternativen gesucht. Bessere Ergebnisse als mit der Maus können beispielsweise mit dem von „Google Earth“ bekannten „Space Navigator“ erzielt werden. Darüber hinaus sind aber auch Spezialentwicklungen für Pathologen im Gange [12]. Das Problem der Geschwindigkeit verliert an Schärfe, wenn eine erweiterte Funktionalität (Parallelpräsentation verschiedener Färbungen, schneller Zugriff auf Archivschnitte, 3 DVisualisierung von Biopsiestufen) in der Summe kürzere Bearbeitungszeiten ermöglicht. Die Primärgeschwindigkeit der VM wird durch den technischen Fortschritt bei Computern und Netzen implizit ansteigen.

\section{Geschwindigkeit der Erzeugung von WSI}

Die Geschwindigkeit der Präparatescanner muss so hoch sein, dass durch ihren Einsatz keine Zeitverluste entstehen.

Hier besteht derzeit noch der größte Handlungsbedarf. Die Entwicklung verläuft allerdings recht schnell, und die Konkurrenz zwischen mehreren Unternehmen treibt die Entwicklung voran. Die Preise fallen, und die Scangeschwindigkeiten steigen an. Das Problem zu geringer Geschwindigkeit kann alternativ durch die Beschaffung mehrerer Scanner gelöst werden, die parallel einzusetzen sind.

\section{Integration in das Pathologie- und Laborinformationssystem}

Das virtuelle Mikroskop muss sich nahtlos in das Pathologie- und Laborinformationssystem integrieren.

Damit ist nicht allein die Integration in ein bestehendes Softwaresystem gemeint, sondern durch die Digitalisierung besteht die Möglichkeit, auch Laborprozesse insgesamt digital abzubilden und als „Geschäftsprozesse“ zu modellieren. Die Standardisierungsorganisationen DICOM, HL7 und IHE befassen sich gegenwärtig mit der Modellierung von Prozessen in der Pathologie in Form von Geschäftsprozessen [13, 14, 15].

Diese Anforderung ist mittelfristig unverzichtbar. Allerdings wird für die Realisierung Zeit benötigt. In der Radiologie erforderte die so genannte RIS-PACS-Integration einige Jahre. Hersteller von $\mathrm{Pa}$ thologie-Informationssystemen und virtuellen Mikroskopen arbeiten bereits intensiv an Lösungen. Hohe Qualität erfordert hierfür eine längerfristige, intensive Zusammenarbeit zwischen Nutzern und Entwicklern.

Die Analyse aller aufgeführten Anforderungen zeigt, dass es keine grundsätzlichen Hinderungsgründe für eine Nutzung der VM in der Routine gibt. Jedoch wird es ein längerfristiger Prozess sein, der eher in Stufen ablaufen wird. Anders als in der Radiologie spielen die erzielbaren Vorteile eine viel größere Rolle. 


\section{Vorteile der virtuellen Mikroskopie}

Aus den oben angegebenen Gründen folgt im Wesentlichen, dass eine erweiterte Funktionalität der entscheidende Antrieb für die Einführung der VM sein wird. Worin liegen jedoch diese erweiterten Möglichkeiten und womit ist zu rechnen? Es folgt eine Aufstellung wesentlicher Entwicklungen.

\section{Optimale Präsentation, Dokumentation des Arbeitsstandes}

Im Kern kann man diese elektronische Fallpräsentation als den „papierfreien Arbeitsplatz" des Pathologen ansehen. All das, was jetzt parallel und in Stapeln vor dem Pathologen und neben dem Mikroskop liegt, lässt sich übersichtlich auf 2 Monitoren anordnen. Auf einem Monitor erfolgt die Darstellung aller relevanten Dokumente, über den anderen erfolgt die Mikroskopie. Tests im Rahmen einer Designstudie haben erhebliche Beschleunigungspotenziale offenbart. Allein die individuell einstellbare, automatische Anordnung von Präparaten in Abhängigkeit von der Fragestellung auf der „elektronischen Klatte“ sowie die Markierung bereits mikroskopierter Präparate und Areale sind einfache, aber effektive Hilfsmittel.

\section{Präparatwechsel und parallele Visualisierung von Färbungen}

Das Wechseln von Präparaten verschiedener Färbungen und speziell die Relokalisation des gleichen Areals im Schnitt benötigt mitunter viel Zeit. Durch VM kann dies „per Knopfdruck“ ohne jeglichen Zeitverzug erfolgen. Voraussetzung dafür ist jedoch, dass für die Schnitte vorher mit einem Computerprogramm eine Registrierung („geometrisches Übereinanderschieben“) erfolgte.

\section{Direkter Archivzugriff}

Unter der Voraussetzung, dass alle Präparate als WSI vorliegen, kann der Zugriff auf die Präparate der Vorbefunde direkt und sofort erfolgen.

\section{Quantifizierung}

Die Quantifizierung von Markern ist ein sehr aktuelles Thema und wird zunehmend als Grundlage für Therapieentscheidungen in der Onkologie eingefordert. Bildanalyse-basierte Verfahren werden bereits in die Zulassung einbezogen $[16,17]$.

Die quantitative Pathologie, wie sie in den 1970er und 1980er Jahren auf vielfältige Weise in der Forschung zum Einsatz gekommen ist, wird möglicherweise ebenfalls eine Renaissance in der Routinediagnostik erfahren. Auf Knopfdruck wird man beispielsweise die Verteilung der Zellkerngrößen in der Invasionsfront eines Tumors mit den analogen Verteilungen verfügbarer Referenzfälle vergleichen können.

\section{Mikroskopierhilfen und Präsentationstools}

Begonnen hat die Entwicklung mit einem jederzeit sichtbaren Übersichtsbild, verbunden mit weiteren Zwischenvergrößerungen. Ein Präparat kann so gleichzeitig mit verschiedenen Vergrößerungen und an verschiedenen Stellen mikroskopiert werden. Allgemein sind auf dem Gebiet der Präsentationstools in naher $\mathrm{Zu}$ kunft die größten Fortschritte zu erwarten. So können etwa Schnittstufen einer Biopsie als virtueller 3 D-Stack sehr komfortabel mikroskopiert werden [18]. Eine automatische Vorverarbeitung zur Erkennung histologischer Strukturen wird die Öffnung eines Falls an einer vordefinierten Stelle und in einer individuell einstellbaren Form ermöglichen.

\section{Fallpräsentation in interdisziplinären Tumorkonferenzen}

In der Regel besteht in diesen Konferenzen die Möglichkeit, per PC und Beamer Fälle zu präsentieren. Durch die Markierung der relevanten Areale im WSI und den direkten Zugriff über das Inter-/Intranet entfallen das Fotografieren und das Erstellen von aufwendigen Präsentationen im Vorfeld. Stattdessen kann bei Rückfragen „live“ mikroskopiert werden. Wir haben erste positive Erfahrungen mit dieser Verfahrensweise gemacht. Kritisch im Hin- blick auf den Zeitbedarf ist allerdings die späte Anmeldung relevanter Fälle durch den Kliniker in Kombination mit längeren Scanzeiten für große Operationspräparate, verbunden mit der Notwendigkeit der zweifachen Mikroskopie - konventionell und virtuell seitens des Pathologen.

\section{Elektronische Fallvorstellung}

Gerade in großen universitären Häusern ist die Abstimmung der Vorstellungszeiten durch die Assistenten bei den Oberärzten immer wieder ein leidiges Problem. Mit der elektronischen „Fallvorstellung“ gibt es hier die Möglichkeit, eine elektronische Warteschlange aufzubauen. Damit können die Anforderungen durch die Oberärzte nach Kriterien wie Dringlichkeit, Aufwand und Zuständigkeit gemanagt werden. Kleine Probleme können ohne Präsenz „telepathologisch“ besprochen werden. Da auf das identische WSI gleichzeitig mehrere Pathologen zugreifen können, sind vielfältige, der jeweiligen Situation angepasste Verfahrensweisen denk- und realisierbar.

\section{Kooperative Diagnostik per \\ Telepathologie - \\ verteilte Pathologie}

Die ursprünglich technisch aufwendige Telepathologie wird zu einer einfachen, in das virtuelle Mikroskop integrierten Funktion. Im Gegensatz zur konventionellen Telepathologie, bei der entweder statische Bilder versendet oder Präparate telemikroskopiert werden, reduziert sich die per VM realisierte Telepathologie auf die Vergabe von Rechten zur Ansicht von Präparaten verbunden mit der Übermittlung des Ortes, an dem das WSI zu finden ist. Darüber hinaus können alle oben genannten Funktionalitäten natürlich auch kooperativ genutzt werden. Es eröffnen sich unkomplizierte Möglichkeiten für eine Zusammenarbeit über geographische Entfernungen hinweg.

\section{Elektronischer Fallvergleich}

Abgeschlossen werden soll diese Aufzählung mit einem Blick in die weitere $\mathrm{Zu}$ kunft. Mit dem Aufbau von Referenzfalldatenbanken besteht die Möglich- 
keit, Vergleichsfälle jederzeit heranzuziehen und diese sogar nach Relevanz vorher ordnen zu lassen. So kann unter Tausenden von Fällen (z. B. aus der eigenen Falldatenbank) nach den ähnlichsten Fällen gesucht und diese wie bei „Google“ in einer „Hitliste“ gemeinsam mit den relevanten Ausschnitten der WSI präsentiert werden. An solchen Systemen wird bereits intensiv geforscht [19].

\section{Ausblick}

Für wen und welche diagnostischen Anwendungen die o. g. Vorteile sich letztlich auszahlen, wird die Entwicklung zeigen [20]. Der vergleichende Blick zur Radiologie ist hier ein Blick in Zukunft: So ist der unmittelbare Zugriff auf Vorbefunde im PACS-Archiv selbstverständlich. Verteiltes Arbeiten in einem teleradiologischen Umfeld ist in großen Kliniken ebenfalls Alltag geworden. Automatische Analyseverfahren halten Einzug in die alltägliche Praxis (z. B. die Detektion von Mikrokalk in Mammographien) und beschleunigen die Diagnostik erheblich.

Auf die interessante Frage, ob eine Einführung der VM für Teilbereiche, bestimmte Aufgaben oder Mitarbeiter sinnvoll ist, gibt die Praxis mittlerweile eine positive Antwort. Im Institut für Pathologie der Charité am Standort Mitte werden WSI beispielsweise für die Präsentation in den Fallkonferenzen der Tumorzentren eingesetzt - nicht immer, aber immer häufiger. Die Präparate zu Konsultationsfällen werden als WSI archiviert, da die Originalpräparate ja wieder an den Einsender zurück gesandt werden. Für die Telepathologie wird zunehmend der T.Konsult Service des Berufsverbandes genutzt, der ursprünglich für die Zweitmeinung im Rahmen des deutschen MammographieScreening-Programms implementiert wurde. An der Semmelweis Universität in Budapest laufen bereits umfangreiche Studien zum Routineeinsatz (B. Molnar, persönliche Mitteilung).

\section{Resümee}

Es wurde hier bewusst nicht detailliert über Scangeschwindigkeiten, Speicherbedarf, Einsparpotenzial und zeitliche Szenarien für die Einführung der VM in

Pathologe 2008 • [Suppl 2] 29:250-254 DOI 10.1007/s00292-008-1093-0

(c) Springer Medizin Verlag 2008

\section{P. Hufnagl $\cdot$ K. Schlüns \\ Virtuelle Mikroskopie und Routinediagnostik. Ein Diskussionspapier}

\section{Zusammenfassung}

Für die virtuelle Mikroskopie (VM) eröffnen sich im Umfeld der Pathologie immer neue Einsatzgebiete. Ausgehend vom Einsatz in Lehre, Weiterbildung und Forschung erfolgen die ersten Anwendungen auch in der Krankenversorgung. Mit den Erfahrungen in der Radiologie und vermehrt in der Pathologie kann man abschätzen, dass sich die VM auch in der Routine etablieren wird. Allerdings wird der Prozess der Einführung anders verlaufen als in der digitalen Radiologie, an deren Anfang der Computer als ", notwendiges Übel" zur Visualisierung von Computertomogrammen per se akzeptiert werden musste.

Virtuelle Mikroskope müssen überzeugen, indem sie die Routinearbeit des Pathologen erleichtern und unterstützen: beim $\mathrm{Ar}$ - chivzugriff, bei der Quantifizierung von Markern, beim Display von Biopsiestufen, bei der Kooperation mit Kollegen usw. Die Frage „wann die VM in der Routine ankommt“, kann nicht allein technisch beantwortet werden. Voraussetzung ist neben einer Scannergeschwindigkeit unter $1 \mathrm{~min} / \mathrm{cm}^{2}$ auch die Finanzierbarkeit. Viel wichtiger ist jedoch die optimale Einbindung der VM in die Alltagsroutine. Dieser Prozess wird sich über Jahrzehnte hin erstrecken, wie die Entwicklung in der Radiologie gezeigt hat.

Schlüsselwörter Virtuelle Mikroskopie · Routinediagnostik . Bildanalyse · Quantitative Pathologie

\section{Virtual microscopy and routine diagnostics. A discussion paper}

\section{Abstract}

There are several areas of application for virtual microscopy in pathology. After broad use in education and research, we are now seeing its initial application in health care. We can predict that, on the basis of experience gained in digital radiology and early experience in pathology, VM will be established as routine. However, its introduction will follow a different course to that taken in digital radiology, which required that the computer be accepted as a "necessary evil" to record and display computer tomograms.

Virtual microscopy needs to ensure that it supports the pathologist significantly in terms of access to archives, quantification of markers, display of biopsy stacks, cooperation with colleagues, etc. Consequently, the question of "When will virtual microscopy enter daily pathology practice?" may not only be answered on the basis of technical features. Of course, it is necessary that scanning speed goes below $1 \mathrm{~min} / \mathrm{cm}^{2}$ and that it remains financially viable, but more important is an optimal integration of virtual microscopy in daily pathology routine. This process will extend over several decades, as past developments in digital radiology have shown.

\section{Keywords} Virtual microscopy - Routine diagnostics . Image analysis · Quantitative pathology 
die pathologische Diagnostik berichtet. Viele der gegenwärtigen Probleme werden durch die technische Entwicklung en passant gelöst, und die Kosten-NutzenRechnung sieht ohnehin für unterschiedlich strukturierte Pathologien anders aus und wird sich abhängig von der preislichen Entwicklung von Jahr zu Jahr ändern. Bereits heute kann man einen 1-TerabyteSpeicher für unter 100 EUR beziehen.

Klar wird allerdings, dass die VM die histologische Diagnostik am Mikroskop in vergleichbarer Art und Weise verändern wird, wie dies durch die Einführung der digitalen Verfahren in der Radiologie bereits erfolgt ist. Genau wie dort wird sich die Entwicklung über Jahrzehnte hinziehen.

Der Quantifizierung von Markern könnte eine Schlüsselfunktion bei der Einführung der VM zukommen.

Hochschulinstitute, die gleichzeitig in Lehre, Forschung und Krankenversorgung engagiert sind, werden analog zur digitalen Radiologie Vorreiter der Entwicklung sein, da sie die größten Synergieeffekte aus der Einführung ziehen können.

\section{Fazit}

Mit der VM wird den Pathologen ein Instrument in die Hand gegeben, welches ihnen die Möglichkeit geben wird, die Herausforderungen, die beispielsweise im Zuge der zunehmenden Einrichtung organbezogener Tumorzentren auftreten, zu meistern. Mit einem überall und sofort möglichen Zugriff auf die histologischen Präparate (Voraussetzung: Computer, Internet-/Intranetzugriff, Browser, Beamer) tritt der Pathologe auch optisch "gleichberechtigt" neben den Radiologen, der "seine" radiologischen Bilder live aus dem PACS demonstriert.

Wie die ersten Erfahrungen mit der VM in der Lehre zeigen, wird dadurch auch das Fach „Pathologie“ für den Nachwuchs wieder interessanter. Gerade für die Gewinnung der bereits mit dem Computer aufgewachsenen Medizinstudenten für das Fach Pathologie ist dies ein nicht unerheblicher Vorteil.

\section{Korrespondenzadresse \\ Dr. P. Hufnagl \\ Institut für Pathologie \\ Charité-Universitätsmedizin Berlin \\ Campus Mitte \\ Charitéplatz 1, 10117 Berlin \\ peter.hufnagl@charite.de}

Interessenkonflikt. Der korrespondierende Autor weist auf folgende Beziehung hin: Er ist Gesellschafter der VMscope GmbH Berlin.

\section{Literatur}

1. Ramirez NC, Barr TJ, Billiter DM (2007) Utilizing virtual microscopy for quality control review. Disease Markers 23: 459-466

2. Tsuchihashi Y, Takamatsu T, Hashimoto Y et al. (2008) Use of virtual slide system for quick frozen intra-operative telepathology diagnosis in Kyoto, Japan. Diagn Pathol (Suppl 1) 3: S6-S6

3. Saeger K, Schmidt D (2006) Digital slide training portal: training slides available on the Internet from the German division of the IAP. Pathologe 27: 477-480

4. Schrader T, Niepage S, Leuthold T et al. (2006) The diagnostic path, a useful visualisation tool in virtual microscopy. Diagn Pathol 1: 40

5. Stewart J 3rd, Bevans-Wilkins K, Bhattacharya A et al. (2008) Virtual microscopy: an educator's tool for the enhancement of cytotechnology students' locator skills. Diagnostic Cytopathology 36: 363-368

6. Schrader T, Beil M, Schmidet D et al. (2007) Virtual microscopy in medical research: Open European Nephrology Science Center (OpEN.SC). In: Horii SC, Andriole KP (eds) Medical Imaging 2007: PACS and Imaging Informatics. Proceedings of the SPIE, vol. 6516. SPIE, San Diego, USA

7. Digital Imaging and Communications in Medicine (DICOM) Standard (2007) Supplement 122: Specimen identification and revised pathology SOP Classes, DICOM Working Group 26, NEMA

8. Kalinski T, Zwönitzer R, Sel S et al. (2008) Virtual 3D microscopy using multiplane whole slide images in diagnostic pathology. Am J Clin Pathol 130: 259-264

9. Demichelis F, Dell'Anna R, Sboner A et al. (2004) Expressionary data from tissue microarray experiments: analysis and graphical representation. Seventh European Telepathology Congress Abstract Booklet. Poznan, Poland, 8-11 July 2004

10. Mager A-K (2008) Die diagnostische Sicherheit und Effizienz der WSI-Telepathologie an Hand des telepathologischen Konsultationsservices T.Konsult Pathologie im Rahmen des Brustkrebsscreening Programms. Dissertation Berlin 2008

11. Forster B, Van De Ville D, Berent J et al. (2004) Complex wavelets for extended depth-of-field: a new method for the fusion of multichannel microscopy images. Micros Res Tech 65: 33-42

12. Pohl S (2008) Virtuelles Mikroskop. Diplomarbeit, Hochschule Magdeburg Stendal

13. Le Bozec C, Henin D, Fabiani B et al. (2007) Refining DICOM for pathology-progress from the IHE and DICOM pathology working groups. Studies in Health Technology and Informatics 129: 434-438

14. Rojo MG, Rolón E, Calahorra L et al. (2008) Implementation of the Business Process Modelling Notation (BPMN) in the modelling of anatomic pathology processes. Diagn Pathol (Suppl 1) 3: 22
15. Schrader T, Beil M, SchaafT (2007) Business process modeling for the information service center for kidney diseases and transplantation (Open European Nephrology Science Center - OpEN.SC). lin: 91. Jahrestagung der Deutschen Gesellschaft für Pathologie (DGP). Magdeburg

16. TriPath Imaging Press Release (2005) TriPath imaging receives FDA $510(\mathrm{k})$ clearance for estrogen receptor and progesterone receptor applications for Ventana Image Analysis System, TriPath

17. Aperio Press Release (2007) Aperio receives FDA clearance for HER2 image analysis application. Aperio

18. Zerbe N (2008) Analyse serieller histologischer Schnitte im Hinblick auf die automatische Bestimmung gleichartiger Partikel benachbarter Schnittstufen. Diplomarbeit, FHTW-Berlin

19. THESEUS-Anwendungsszenario MEDICO: http:// www.theseus-programm.de/scenarios/de/medico

20. Glatz-Krieger K, Glatz D, Mihatsch MJ (2006) Virtuelle Mikroskopie. Erste Anwendungen. Der Pathologe 6: 469-476 